\title{
Magnitude dependence of radiated energy spectra: Far-field expressions of slip pulses in earthquake models
}

\author{
Bruce E. Shaw \\ Lamont-Doherty Earth Observatory, Columbia University, Palisades, New York, USA \\ Received 25 July 2001; revised 11 July 2002; accepted 18 October 2002; published 18 February 2003.
}

[1] We examine the radiated waves emitted by events on a model fault. The model deterministically produces a complex sequence of events, with a wide range of sizes, from a uniform frictional instability. The spontaneous rupture events emit a rich spectrum of radiated waves as they nucleate, propagate, and decelerate within the complex stress field left by previous events. Two model innovations, a new driving boundary condition on the fault and a new radiating boundary condition which allows a spatially varying prestress away from the fault, allow us to directly measure the radiation without problems from boundary reflections in our two-dimensional model. We quantify the radiation by first measuring the energy spectral density and then averaging over events of a similar size to examine the magnitude dependence. Assuming only a physics of the tractions on the fault, we obtain a full spectra of radiated waves for a complex population of events with a wide range of sizes. To quantify the resulting spectra, we consider two different spectral measures. One, the peak amplitude of the spectral energy density, occurs at a period which scales with the rupture length and corresponds with the classical corner frequency measurement. The other, the peak amplitude of the spectral average acceleration or the low-frequency corner in the case of a flat acceleration spectrum, occurs at a period that scales with the duration of slip of points on the fault. The period of the peak spectral acceleration saturates for large events. Looking at the rupture motions on the fault, we find that this spectral behavior corresponds with the behavior of slip pulses in the model. Intense narrow pulses of slip develop for very long rupture events. We quantify this by measuring the mean slip duration as a function of rupture length and show that it is has the same behavior as the peak period of spectral acceleration. Thus the duration of the slip pulses in these ruptures is directly expressed in their radiated spectra. Moreover, we find that these corner periods exhibit a nontrivial dependence on event magnitude for the different frictional instabilities that we have examined, suggesting that any observed dependence of these corner periods on earthquake magnitude might provide insight into the frictional instability of earthquakes. INDEX TERMS: 7212 Seismology: Earthquake ground motions and engineering; 7260 Seismology: Theory and modeling; 7209 Seismology: Earthquake dynamics and mechanics; 7215 Seismology: Earthquake parameters; KEYWORDS: earthquakes, spectra, magnitude dependence, corner frequency, slip duration, slip pulses

Citation: Shaw, B. E., Magnitude dependence of radiated energy spectra: Far-field expressions of slip pulses in earthquake models, J. Geophys. Res., 108(B2), 2100, doi:10.1029/2001JB000741, 2003.

\section{Introduction}

[2] The waves radiated by earthquakes are perhaps the most important earthquake behavior: they cause nearly all the damage, and, further, they carry with them the most intimate views of the source dynamics we have. Despite their central importance, they remain incompletely understood, as there are a number of factors complicating the observations. First, attenuation convolves the emitted radiation with uncertain path- and frequency-dependent effects. Second, sparse measurements of infrequent large events

Copyright 2003 by the American Geophysical Union. 0148-0227/03/2001JB000741\$09.00 give poorly sampled data sets. Finally, the poorly understood dynamics and the lack of good first-principles theoretical models to guide our understanding leave us with few tools to help explore what we might see in the data and to formulate new questions to ask of it.

[3] This paper presents a new tool in our search to understand the radiation emitted by earthquakes and the underlying physics it reflects. We present a dynamic model capable of generating events with a wide range of sizes, with richly complex waves emitted by the events, and a minimum of assumptions in the model. Having assumed only a constitutive law for the tractions on the fault, we obtain the full spectral content of the radiated waves for a complex population of events with a wide range of sizes. 
Averaging over events of a similar size, we measure the farfield radiated energy spectra as a function of rupture length. This is a quantity of fundamental importance to earthquake hazard. We go further by exploring the dependence of the radiated energy spectra on characteristics of the spontaneous ruptures. Exploring a range of frictional instabilities, including slip- and velocity-weakening friction, as well as timedependent friction, we find nonlinear scaling of the period of the peak of the radiated average acceleration spectra with magnitude. Examining the rupture motions on the fault, we find the peak period for the radiated average acceleration spectra scales with the mean slip duration of points on the fault, for moderate and large events where the slip and velocity weakening stress drop mechanisms are dominant. These means, again, are found to scale in nontrivial ways with event size.

[4] Pioneering work by [Haskell, 1964] generated theoretical curves for the radiated energy spectra from a moving finite dislocation source, and further related the resulting energy spectral density to fault width and slip duration. A great amount of effort has continued this approach of specifying source motions and calculating the resulting radiation. While this approach has the virtue of often allowing closed form solutions with which one can examine the influence of various source motions, it has the limitation of being a kinematic rather than dynamic approach, and thus the physical relevance of the solutions and the underlying physics which would generate such solutions remains unresolved.

[5] Previous theoretical work on generating magnitudedependent radiated spectra has generally begun from assumed scaling laws inferred from earthquake observations $[$ Aki, 1967; Brune, 1970] and then attempted to match them through various combinations of assumptions about source motions, rupture propagation, and distributions of heterogeneities [e.g., Hartzell et al., 1999; Tumarkin et al., 1994; Zeng et al., 1994]. There is, however, a fundamental limitation with this approach, which is that it does not tell us what spectra we should expect to see. Also, given that some controversy has developed [Boatwright and Choy, 1992; Atkinson and Silva, 1997; Haddon, 1996; Atkinson et al., 1997; Haddon, 1997; Sokolov, 1997] over whether there may be observed deviations from the idealized classical spectra [Brune, 1970], a derivation of the spectra from first principles is clearly important. Our work is new in that we have achieved this, having had to assume only the nature of the friction on the fault.

[6] There is a long history of work relating source motions to radiated energy [Madariaga, 1976; Miyatake, 1980; Das and Kostrov, 1988; Olsen et al., 1997]. Our work is new in pointing to a feature of the radiated energy which is observable and may have a nontrivial behavior, the magnitude dependence of the period of the peak of the radiated average acceleration spectra, and how that may reflect nontrivial scaling of the mean slip time with rupture size. In previous work, additional corners in the frequency spectra have been reported and debated [Boatwright and Choy, 1992; Atkinson and Silva, 1997; Haddon, 1996; Atkinson et al., 1997; Haddon, 1997; Sokolov, 1997]. A number of possible physical origins of a second corner have been discussed, including partial stress drops [Brune, 1970], and various irregularities [Hartzell and Brune, 1979;
McGarr, 1981; Papageorgiou and Aki, 1983; Joyner and Boore, 1986; Boatwright, 1988; Atkinson, 1993; Atkinson and Silva, 2000]. Our work finds another origin: the development of slip pulses.

[7] Finally, one significant application of our work is the scaling of spectra radiated by large and great earthquakes. This question has received some previous attention [Haskell, 1964; Papageorgiou and Aki, 1983; Atkinson and Silva, 2000], but this work substantially extends our theoretical tools by generating dynamically consistent results across the full range of sizes.

[8] The rest of the paper is organized as follows. In section 2 , we discuss the model and present two model innovations which allow us to directly measure the radiated energy, a new loading traction on the fault, and a new radiating boundary condition away from the fault. In section 3, we present the measurements of the radiated energy spectra and measurements of the source motions which explain features of the measured radiated energy spectra. In section 4, we discuss some further issues and recast some of the results for better comparison with observations. Appendix A specifies the friction used in this paper. Appendix B presents the new radiating boundary condition. Appendix $\mathrm{C}$ discusses the far-field asymptotics of the two-dimensional (2-D) radiation.

\section{The Model}

[9] Because we are concerned with average properties spanning a wide range of sizes of events, we need large numbers of events. To obtain large numbers of events with a high degree of spatial resolution, we study lower dimensional models, which have a huge speedup numerically. We examine a uniform scalar two-dimensional model. This has the minimum parameterization, being uniform spatially and being the minimum dimensionality allowing radiation. We study this simplest model as it contains the basic behaviors we wish to explore, has the fewest parameters which need to be specified, and is fast enough to handle our demanding problem. There will, of course, be quantitative differences with the full three dimensional tensor problem. We expect, however, that the qualitative effects that we present here will nevertheless carry over to the full case.

[10] Our model is an extension of a previous two-dimensional scalar elastodynamic model [Shaw, 1997] with two new innovations. First, we add the driving to the fault itself, rather than to a boundary away from the fault. This allows us to maintain the wave equation in the bulk, and the effect of the loading of the fault by the stably sliding lower fault, while at the same time moving the far boundary away so as to extend the wave equation domain of the bulk. Thus rather than a strip of the wave equation as before, we now have a model which works in a half-space. Since we model the bulk explicitly, in practice, we need to operate in a finite domain. Our second innovation is a slight but important modification of the radiating boundary conditions of Clayton and Endquist [1977] so that they work with spatially varying prestress. Together, these two innovations allow us to measure directly the radiation emitted from the fault and track it for a long time, with only very small perturbations from the far radiating boundary. 


\subsection{Equations for the Model}

[11] In the bulk, we have the wave equation for the displacement $U$

$$
\frac{\partial^{2} U}{\partial t^{2}}=\nabla^{2} U
$$

where $t$ is time, $\nabla^{2}=\partial^{2} / \partial x^{2}+\partial^{2} / \partial y^{2}$ is the twodimensional Laplacian operator for the direction $x$ which is taken to be parallel to the fault and $y$ the direction perpendicular to the fault. We use dimensionless units throughout to minimize the number of parameters. Here we have set the speed of sound to unity. On the fault, located at $y=0$, the tractions $\mathcal{T}$ balance the strain

$$
\left.\frac{\partial U}{\partial y}\right|_{y=0}=\mathcal{T} .
$$

The tractions consist of two parts, a friction $\Phi$, which we will discuss shortly, and the coupling to the stable sliding plate displacement

$$
\mathcal{T}=\Phi+\kappa(\nu t-U)
$$

Here $v \ll 1$ is the slow plate velocity, and $\kappa$ is a coupling constant with dimensions of inverse length scale. The physical origin of this second coupling traction is the connection elastically, in the full three-dimensional problem, of the upper unstably sliding fault to the lower stably sliding fault, which moves at the plate rate $v$. In collapsing the depth dimension to go to two dimensions, this would give a stress which would depend on the relative displacement, with a stiffness $\kappa$ inversely proportional to the loaded seismogenic zone $W$ (a length scale of order $15 \mathrm{~km}$ in strike-slip environments and $60 \mathrm{~km}$ in subduction zones).

[12] One approximation of this coupling term relative to the full 3-D effect is it neglects the retarded aspect of the interaction; here the coupling is instantaneous to the relative displacement, rather than delayed by the time it would take the wave to travel down and back, a time $2 \mathrm{~W} / \mathrm{c}$, where $c$ is the wave speed. We experimented with various ways of mimicking this retarded effect, to see if it might be important, and it did not appear to be. One alteration we examined was to change the $U$ term in the traction to one with some memory, replacing in equation (3)

$$
U \rightarrow \int_{-\infty}^{t} U\left(t^{\prime}\right) e^{-\tilde{\gamma}\left(t-t^{\prime}\right)} d t^{\prime}
$$

so it takes a finite timescale $1 / \tilde{\gamma}$ to adjust to its asymptotic steady state value. The behaviors we examined did not appear sensitive to this alteration. So, in the spirit of minimal parameterization, we dropped it.

[13] This loading of the unstably sliding upper fault by the stably sliding parts of the crust is an essential part of the physics of faults. One previous two-dimensional model incorporated it through coupling though the bottom of the crust, giving a Klein-Gordon equation in the bulk [Myers et al., 1996]. This has the advantage of allowing for a halfspace but the disadvantage of introducing intrinsic dispersion in the bulk. Another previous model incorporated it

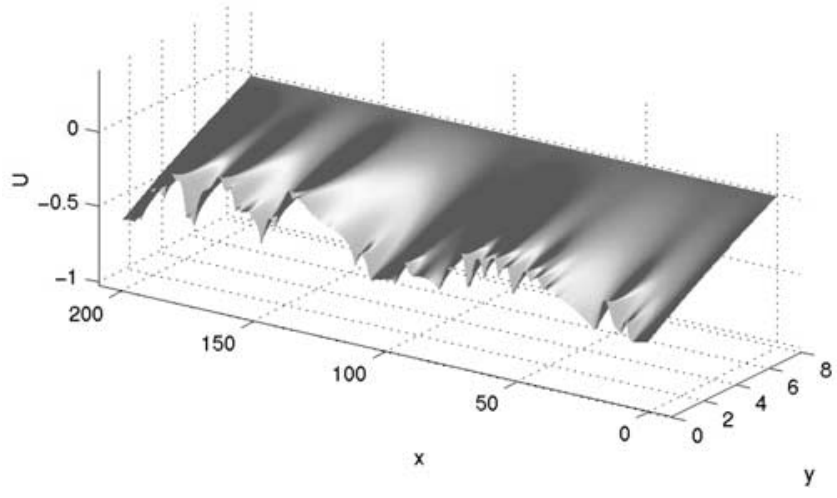

Figure 1. Surface plot of displacement $U$ on the fault, at $y=0$, and in the bulk, for $y>0$. For this stuck static configuration the bulk smoothly interpolates from the boundary, being a harmonic solution of the Laplacian.

through the coupling to a nearby (a crust depth away) surface sliding at the plate rate [Shaw, 1997]. This has the advantage of allowing the wave equation in the bulk, which has the proper dispersion properties and includes the retarded aspect of the interaction. However, it has the disadvantage of introducing a nearby stiff boundary which produces huge reflections and distorts the radiated field. Our new representation of this effect through the additional traction yields a dispersionless bulk of arbitrary width.

[14] The friction used, which is the central core of the complex behavior of the model, has been used and presented before; thus we briefly summarize it, for completeness, in Appendix A. The other boundary conditions are as follows. Properties are completely uniform along the fault, and to maintain this minimal situation, we use periodic boundary conditions in the direction along the fault:

$$
U\left(x+L_{x}\right)=U(x)
$$

[15] Away from the fault, we use a radiating boundary condition during dynamic rupture. Between events, we quench the system to a static solution with the far boundary fixed at the plate displacement. Appendix B discusses the motivation for treating this boundary this way, and the innovative radiating boundary condition we have used. Its main effect is to mimic what we would get with a distant transparent boundary moving at the plate speed.

[16] Figure 1 illustrates the geometry of the problem, with a solution of the displacement in the stuck configuration, when $\partial^{2} U / \partial t^{2}=0$, so the bulk satisfies Laplace's equation.

\subsection{Numerical Simulation}

[17] We discretize our equations, using a second-order finite difference scheme, into lengths $\delta_{x}$ along the fault and $\delta_{y}$ perpendicular to it. There are, necessarily, dispersion effects at high wave numbers with finite difference approximations [Alford et al., 1974]. However, by focusing our measurements on quantities like absolute values of amplitudes of the energy spectra, which do not depend on phase information, we can push the measurements out to higher frequencies than would normally be possible.

[18] Beginning from any nonuniform initial condition, the system rapidly evolves to an attractor. For frictional strength- 

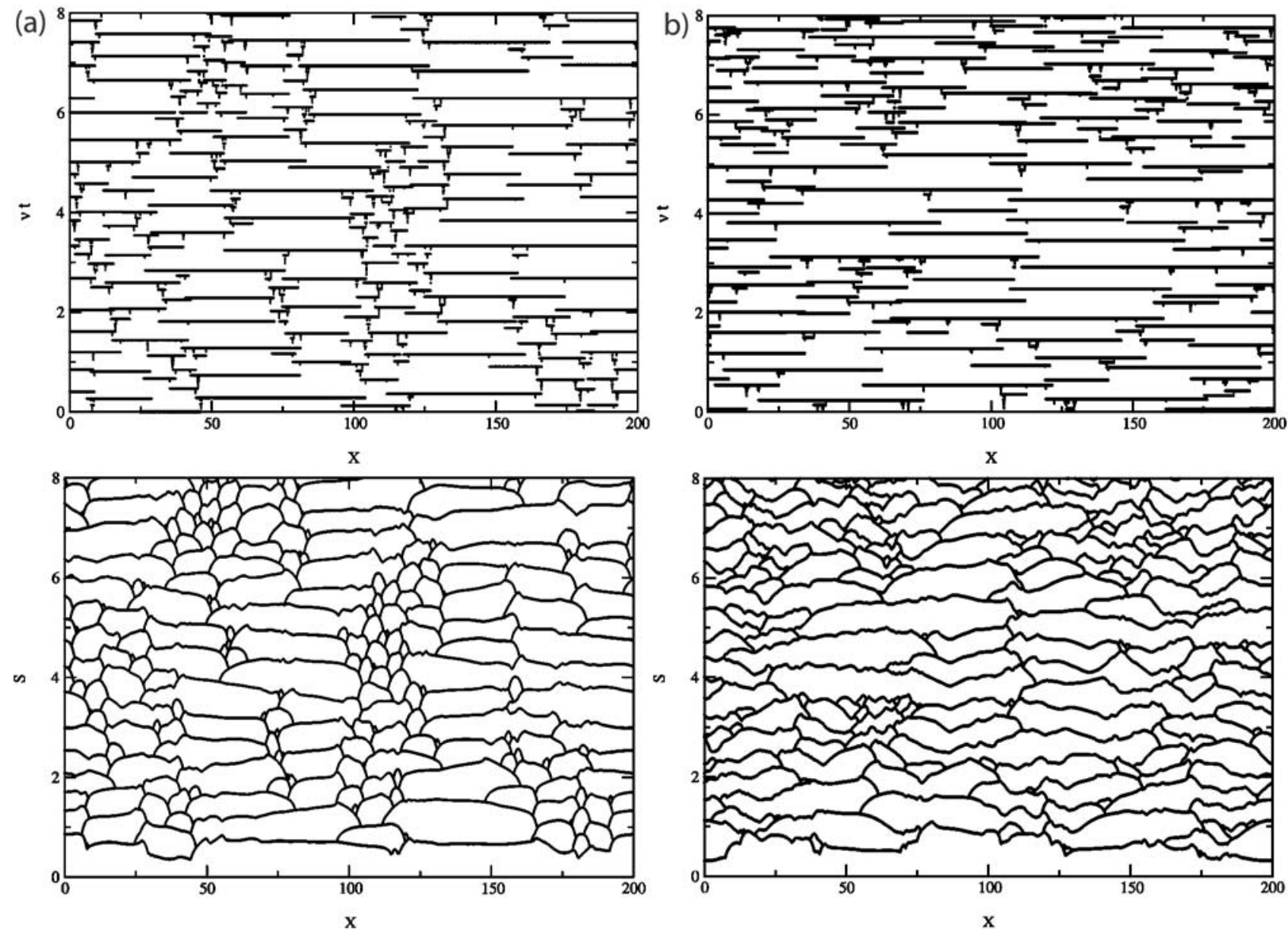

Figure 2. Representations of the attractors for two different frictions with (a) slip weakening and (b) velocity weakening. Two different representations of the attractors in each case are shown. (top) Times at which events along the fault slipped. (bottom) Cumulative slip along the fault following events. For each of the bottom representations, the same events are shown in the top and bottom representations.

ening, the attractor is trivially periodic. In contrast, for frictional weakening, complex attractors can emerge. For this friction, there is a generic large event complexity; for long enough faults and large enough weakening a spatiallytemporally complex irregular population of aperiodic large events is seen. There is, in addition, a relatively narrow range of parameters over which small event complexity is also observed [Shaw and Rice, 2000]. This small event complexity, when it exists, exhibits power law distributions of event sizes, and reflects a very richly complex attractor in that region of parameter space. While narrow, in terms of parameter space, the small event complexity is nevertheless a legitimate continuum behavior [Shaw and Rice, 2000] and not due to discreteness [Rice, 1993]. Because we are interested in exploring a wide range of event sizes, we focus our attention on the range of parameters where small events occur. This parameter range is where the frictional weakening is close to, but slightly stronger than, the elastodynamic destressing due to sliding. For slip weakening this is related to the strain from the coupling to the stably sliding lower layer, while for velocity weakening it is related to the radiation damping. Figure 2 a illustrates two views of the complex attractor for the slip weakening case near the critical frictional weakening value where both small and large events are seen. Figure $2 \mathrm{~b}$ illustrates a velocity-weakening case, again near the critical value where there are small events.

[19] The results we will discuss are relatively insensitive to parameter values, being swamped by the larger scaling issues as we will see. Changing frictional parameters changes the results quantitatively only slightly and qualitatively not at all. The main effect of operating near the critical weakening values are the fact that the small and intermediate magnitude events occur.

[20] The canonical set of parameters that we will perturb are as follows: For the bulk, $L_{x}=200, L_{y}=8, \delta_{x}=1 / 16, \delta_{y}=$ $1 / 32$. For the friction (see Appendix A for definitions of parameters), $\kappa \equiv 1, \eta=0.003, \sigma_{0}=0.01, t_{0}=0.1, \sigma_{\beta}=0.1$, with, for slip weakening, $\alpha=3, \gamma=0.1, \beta=10$, and for velocity weakening, $\alpha / \gamma=3, \gamma=3, \beta / \gamma=10$. Again, none of the results which follow appear to be sensitive to these parameter choices.

\section{Radiation}

[21] We begin our examination of the radiated field by looking in the time domain. Figures 3 and 4 show the time 

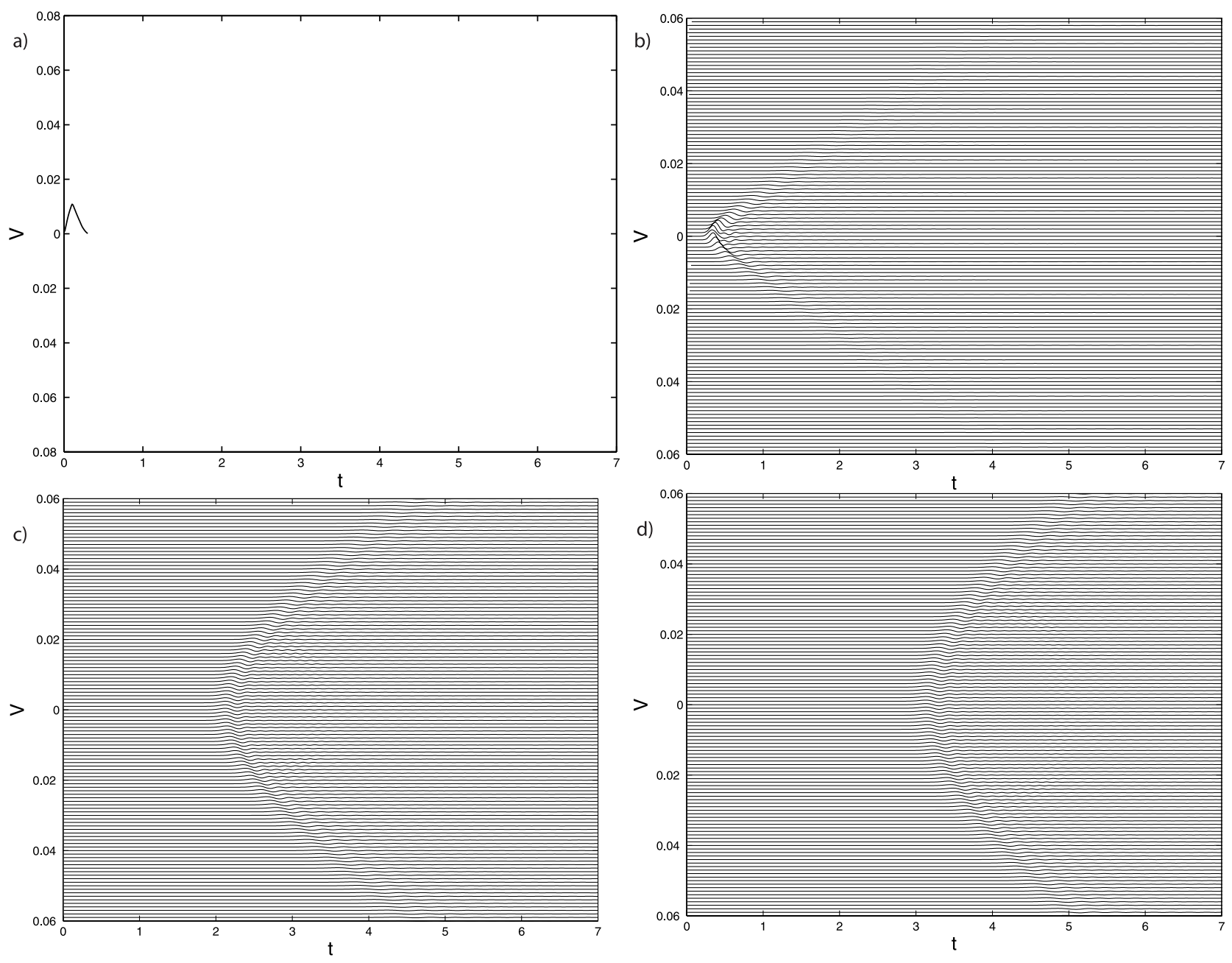

Figure 3. Array of velocity records for different distances from fault. This is a one element event, showing a kind of “empirical Green's function" for the model. The vertical axis is velocity, while the horizontal axis is time. Neighboring records are offset in the vertical direction by a constant velocity increment, to more easily see the correlations. From left to right, from top to bottom, the distances of the arrays from the fault are $\Delta_{y}=0,0.25,2,3$, respectively. Thus the top left panel shows the motions on the fault, and the other panels show the radiated waves at increasing distances from the fault.

series for example small and large sized events. The small event in Figure 3 is the smallest of the events, being only one element $\delta_{x}$ long, and shows effectively an empirical Green's function for the problem. The large event in Figure 4 begins bilaterally, with one side dying out early and the other propagating further before dying out. We set up an array of velocity meters at a fixed distance from the fault, equally spaced from each other, and plot a stack of the resulting measured velocities. Each panel is for a different distance from the fault, beginning on the fault, and moving progressively farther away. The distances of the array are $\Delta_{y}=0$, $0.25,2,3$ away, and illustrate the motions on the fault, and the radiation emitted moving from the near to the far field.

[22] In Figure 4 we can also see that the radiating boundary condition is working quite well. The radiating boundary is located at $L_{y}=4$, and thus reflections would begin to show at the array at times $2 L_{y}-\Delta_{y}$. The clear absence of any noticeable reflected energy in the bottom panels, at times 6 and 5 for $\Delta_{y}=2$ and 3 , respectively, shows that the boundary condition is working.
[23] The large event in Figure 4 shows strong directivity, with the energy focused in the direction of the propagating rupture. We also see how irregularities in the slip due to irregular prestress radiate strongly into the bulk. We again see the radiating boundary condition is working well, even at these large amplitudes, through the lack of any noticeable reflected energy.

[24] An alternative picture of the events can be obtained by analyzing them in the frequency domain. If we take a Fourier transform of the velocity records and look at the square of the amplitudes, we obtain a physically relevant representation of the motions, as these squared amplitudes are the energies in each frequency band. This representation then provides a natural basis to sum, as well as average, since we are summing physical quantities obeying conservation laws, energy.

[25] Thus, by placing an array of meters a fixed distance from the fault and summing over the energies measured across the whole array, we can measure the energy flux through a plane (a line in our 2-D model) a given distance 

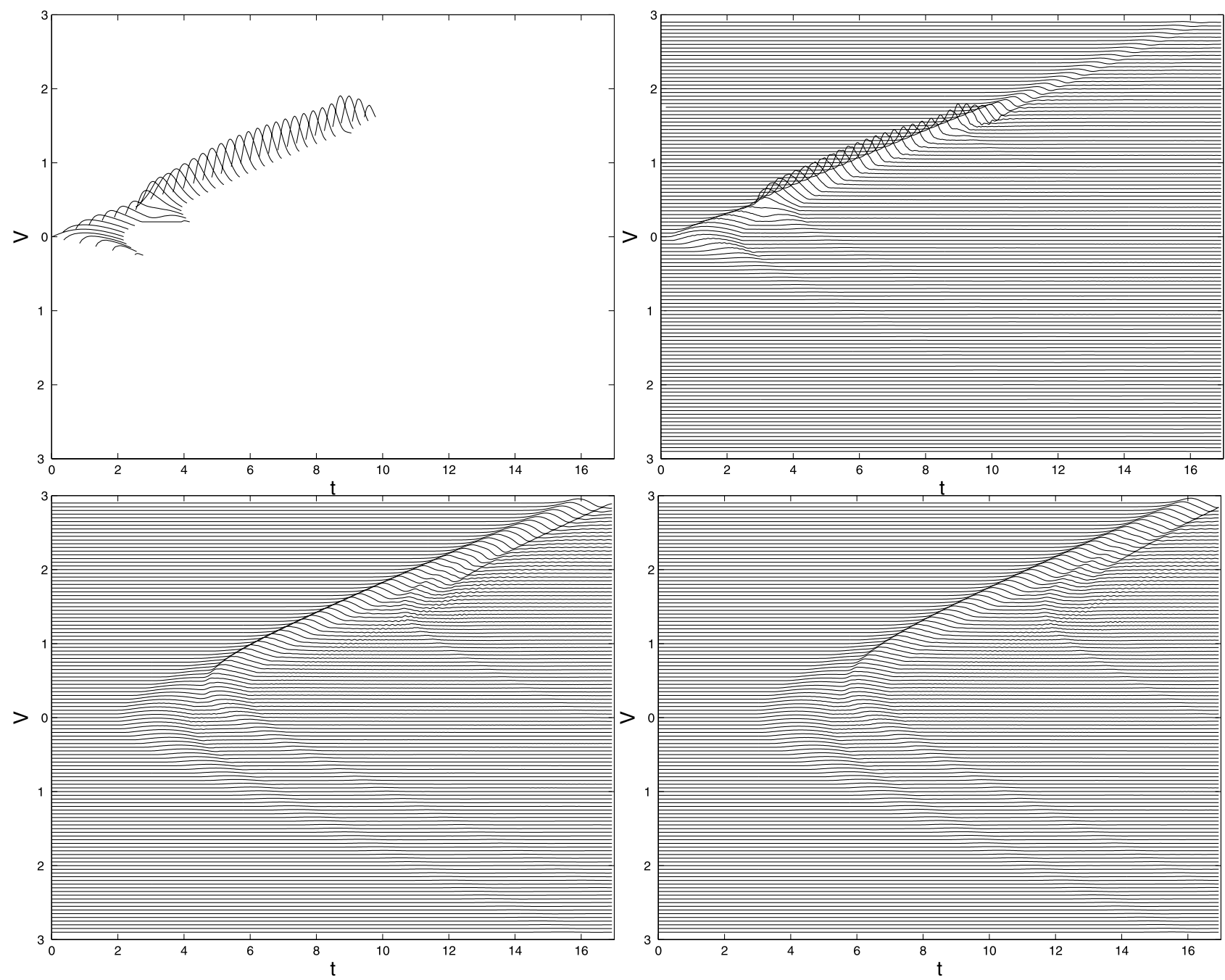

Figure 4. Array of velocity records for different distances from fault. This is a large event in the model, with a nearly unilateral rupture. Note the large-scale change relative to the previous small event in Figure 3. The vertical axis is velocity, while the horizontal axis is time. Neighboring records are offset in the vertical direction by a constant velocity increment, to more easily see the correlations. From left to right, from top to bottom, the distances of the arrays from the fault are $\Delta_{y}=0,0.25,2,3$, respectively. Thus the top left panel shows the motions on the fault, and the other panels show the radiated waves at increasing distances from the fault. For the fault motions in the top left panel, we see a unilateral rupture with varying slip and varying rupture velocity. The varying slip can be seen by the varying areas under the curves, which are the slip. The varying rupture velocity can be seen by the varying slopes between when neighboring parts of the fault initiate slip.

from the fault. We show such a measurement in Figure 5 for the small event shown in Figure 3, for various distances from the fault. Specifically, what we plot in Figure 5 is

$$
E_{\omega}(y)=\int \frac{1}{2}\left(\int \frac{\partial U(x, y, t)}{\partial t} e^{i \omega t} d t\right)^{2} d x
$$

with the corresponding period $T=2 \pi / \omega$ plotted in Figure 5 . Thus, in Figure 5 we see how the spectrum of the radiated waves changes as we move from the near to the far field. Note that from Parseval's theorem,

$$
\int \mathrm{v}^{2}(t) d t=\int \hat{\mathrm{v}}^{2}(\omega) d \omega
$$

so the area under each curve is the total radiated energy. As we move from the near field to the far field, the longer wavelengths are absorbed; that is, they are transferred from kinetic energy to potential energy. As we move away from the fault, the spectra approach an asymptotic form, the farfield spectrum of the radiation. Because of its fundamental significance, we will focus our attention on this asymptotic far-field spectrum.

[26] We can test our numerical measurements of the spectra against calculations for the simplest one element event. In Appendix $\mathrm{C}$, we derive the expected scaling of the spectra at low frequencies with distance, showing $\hat{V}(\omega) \sim \sqrt{\omega / r}$. The linear increase of $\hat{V}^{2}$ with $\omega$ matches the asymptotic low-frequency spectra well, as the dotted line linear in $\omega$ illustrates. The $1 / r$ effect does not show up in the asymp- 


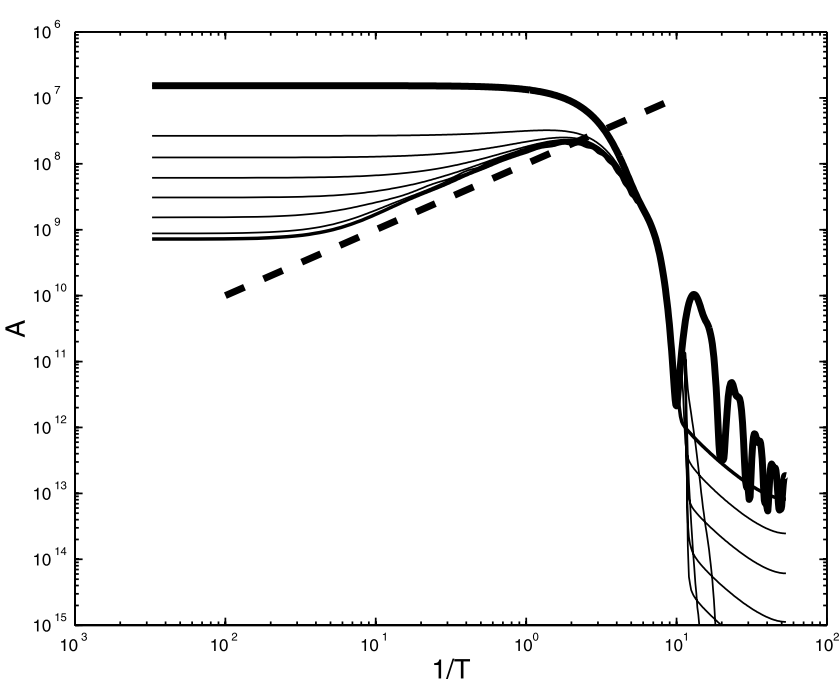

Figure 5. Spectral energy density for one block event in Figure 3, moving from source to far field. Note approach to far-field asymptotic linear growth in $\omega=2 \pi / T$ for small $\omega$, indicated by fit to dashed line with slope of unity.

totic spectra, since we integrate along $d x$ and thus enclose the fault. It does show up, however, in the near-field corrections to the low-frequency amplitude, which scale as $1 / \Delta_{y}$ : Figure 5 shows that as $\Delta_{y}$ increases by a factor of 2, $\hat{V}^{2}(\omega=0)$ decreases by a factor of 2 . Thus the spectral shape at low frequencies (how close we are to a linear increase of $\hat{V}^{2}$ with $\omega$ ) gives us a measure of how close we are to the far-field asymptote.

[27] It is important to reiterate that we do not have any intrinsic dissipation or scattering in our conservative bulk. The changes we see in the spectra in the near field are due to the conversion from kinetic to potential energy, rather than loss by dissipation. This approach to the asymptotic state in the far field also reflects the conservative nature of the bulk. Adding dissipation to the bulk alters this picture by absorbing the radiation; frequency-dependent dissipation would progressively distort the spectrum with increasing distance from the source. In the Earth, there is small but significant dissipation, which most strongly affects the high frequencies. Because of the uncertainties in detail of this dissipation, disentangling from the measured spectra the source and the path effects remains an area of active research. Here we examine the idealized case where there is no dissipation, so that our signal is completely a source effect.

[28] One numerical point is that while we do not have dissipation in the bulk, we do have dispersion in the bulk, due to the finite resolution of our finite difference approximation of the continuum. These effects become significant at the highest frequencies, with the highest frequencies being substantially slowed [Alford et al., 1974]. However, by considering the amplitudes of the waves we avoid this distortion of the wave field and obtain accurate measurements of the energies out to higher-frequency bands than would normally be considered accurate for phase-sensitive measurements, as long as we wait long enough for the waves to arrive.

[29] Figure 6 shows the spectra for the large event shown in Figure 4, again moving from the near field outward. We see that for this larger event it is difficult to get far enough away to be in the far field for the lowest frequencies, evidenced by the lack of a linear $\omega$ asymptote. Nevertheless, from an energetics point of view, we get a good measurement, with a well-defined peak at finite frequencies.

[30] If we sum the energy over the frequencies as in equation (7), there will be very little contribution from these lowest frequencies. Using conservation of energy, we can calculate the total radiated energy by looking at the difference in potential energy before and after the event, and subtracting the dissipation on the fault [Shaw, 1998]. For our case here, where there is an additional traction based on the difference in displacements which stores energy, the $\kappa$ term in equation (3), we need to keep track of the boundary strain energy implicit in this term, as well as the bulk strain energy. Doing the bookkeeping, we are rewarded with an estimate of the total radiated energy that is independent of the energy flux measurements near the fault. It is a check on our numerical methods that these two independent measurements indeed are consistent with each other.

\subsection{Average Spectra}

[31] Having considered these far-field spectra, we now look at the average behavior across a wide range of event sizes. Sorting events into groups of similar size, we average the spectra, obtaining the average spectra as a function of rupture size (here, grouped according to rupture length). We calculate the mean $\bar{E}(\omega, L)$ spectra, where the average is over events having lengths between $L$ and $\lambda L$, with $\lambda>1$ a bin size scaling factor; $\left.\bar{E}(\omega, L)=\langle E(\omega)\rangle_{\left(L^{\prime} L\right.} L_{L^{\prime}}<\lambda L\right)$, so we consider logarithmic bins in length. We sum each spectra over a line a fixed distance $\Delta_{y}$ from the fault, to get a total radiated spectra, and then average the resulting spectra. Thus we obtain our first principal result, the energy spectra radiated by a range of sizes from the smallest events with lengths of only a small fraction of the crust depth $W$, to the largest events breaking tens of crust depths in length. Figure 7 shows the resulting average spectra for the two

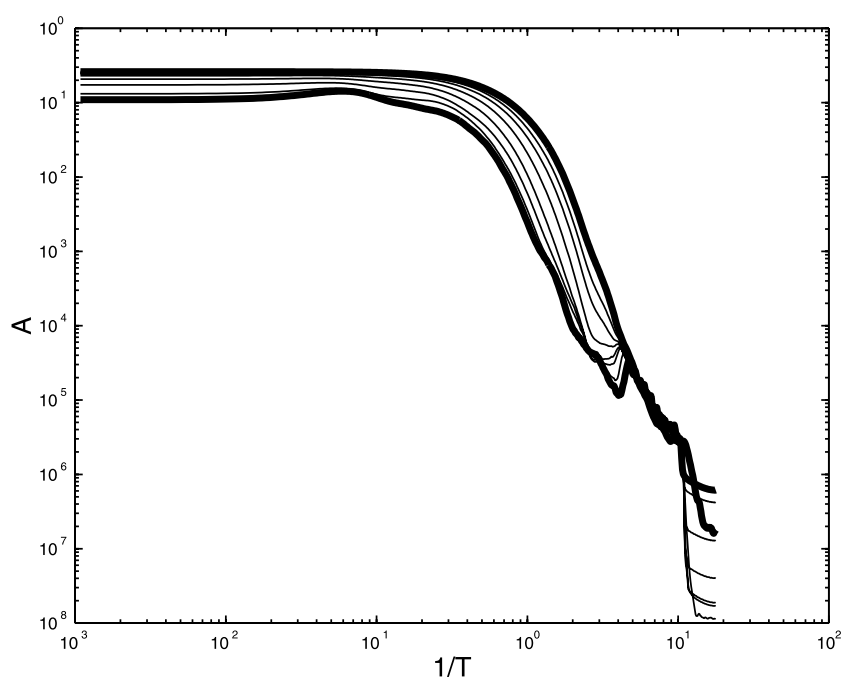

Figure 6. Spectral energy density for large event in Figure 4 , moving from source toward the far field. Note that we have not yet reached the far-field spectra, evidenced by the lack of a linear growth at low frequencies $1 / T$. 

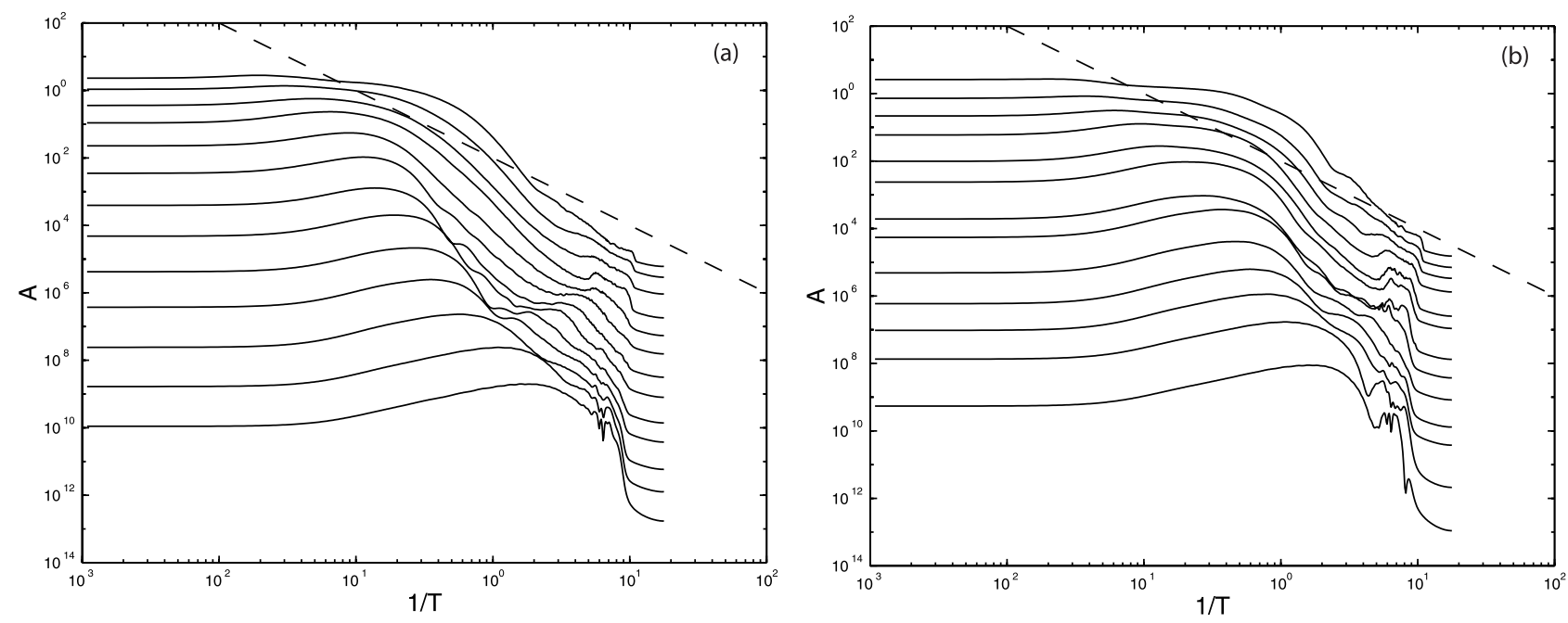

Figure 7. Average spectral energy density for events of different sizes. (a) Slip weakening $(\gamma=0.1)$. (b) Velocity weakening $(\gamma=3)$. Dashed line has slope $\omega^{-2}$.

cases of slip-weakening and velocity-weakening friction. There are a number of interesting aspects to this plot. First, both types of frictions look qualitatively similar, though the radiation is shifted to higher frequencies for the velocityweakening friction relative to the slip-weakening friction. Second, the mean energies at all frequencies monotonically increase as the events get larger. However, the increase occurs in a nontrivial way, in that the curves are not simply scaled versions of one another.

[32] From a comparison of the slope of the spectra at frequencies below the peaks with the far-field asymptotic linear $\omega$ calculation (equation (C7)), we can see that we are close to the far-field spectra for the small events, but not for the largest events. These measurements were carried out at $\Delta_{y}=6$. The binned lengths corresponding to the different curves, from bottom to top, are $L=0.06,0.10,0.17,0.28$, $0.46,0.76,1.3,2.1,3.4,5.6,9.3,15.3,25.2,41.6$. From the slopes we see the approximately far-field spectra persist to $L \approx 5$, a length of order the distance $\Delta_{y}$ from the fault. While the larger events are not in the far field, we will see that we can still quantify some of the features of the spectra relevant to the far field. For example, all but the very largest events have peaks in the energy density at finite frequencies.

[33] At the high frequencies a number of effects need to be considered. At the very highest frequencies, we are limited by finite resolution, and the waves do not propagate. The Nyquist frequency for the discretization that we use here of $\delta_{x}=1 / 16$ and $\delta_{y}=1 / 32$ gives an upper bound of $1 / T \approx \delta_{y} / 2=16$. Dispersion slows wavelengths close to these values [Alford et al., 1974], giving cutoffs of around half this resolution in practice. At frequencies below these cutoffs, we see the source excitation directly. The peaks around $1 / T \sim 5$ are associated with the stress drop $\Sigma$. For the smallest events the time-dependent drop $\sigma_{t}$ dominates; changing the timescale of the drop $t_{0}$ changes the location of the peak, and changing the strength of the drop $\sigma_{0}$ changes the amplitude of the peak. For the moderate and large events, the stress drop $\sigma_{\beta}$ dominates; changing $\beta$ and $\sigma_{\beta}$ have analogous effects in shifting the location and amplitude of the peak, respectively. Thus details of the stress release dominate the highest frequencies. Nevertheless, there are, as we will see, some general features of the spectra we can measure to gain some useful information.

\subsection{Corner Periods}

[34] The first measurement we make is to measure the period $T_{e}$, where the peak amplitude of the radiated energy spectrum occurs. The top solid curves in Figure 8 show how $T_{e}$ scales with rupture length $L$. We see an approximately linear growth of $T_{e}$ with rupture length, corresponding to the classical rupture length corner frequency effect. The different curves in Figure 8, corresponding to different values of $\gamma$, all show the same behavior; thus this effect is quite insensitive to the friction, holding for slip weakening as well as velocity-weakening friction.

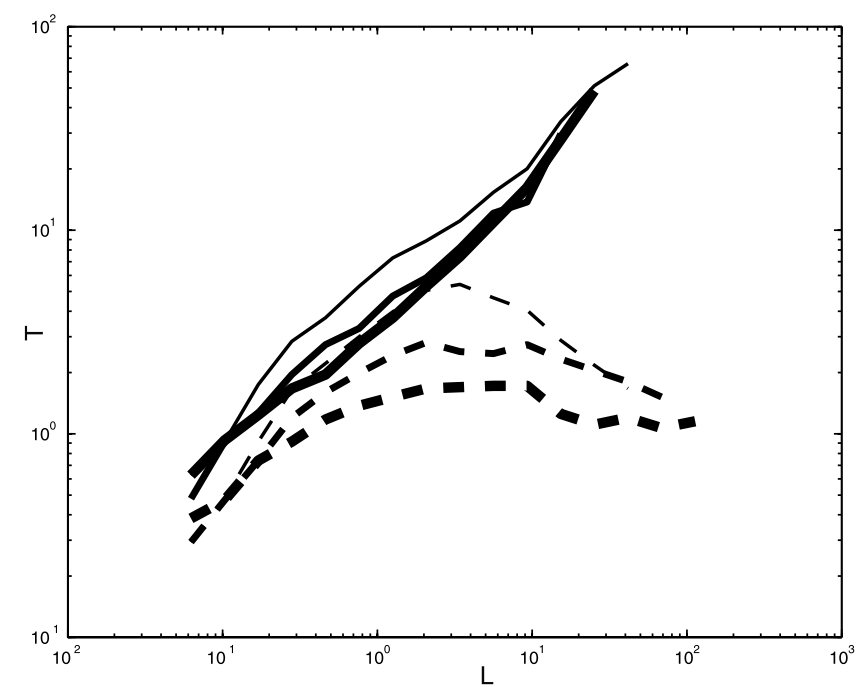

Figure 8. Two corner frequencies for spectra as a function of rupture length $L$. The different thickness curves correspond to different frictional parameter $\gamma=0.1,1,3$, with the thicker lines corresponding to larger values of $\gamma$. The top solid curves are the period of the peak energy density, while the bottom dashed curves are the period of the peak-averaged acceleration spectra. 
[35] In addition to energy, another important measure is acceleration, with acceleration being strongly linked to damage. We can transform our energy spectra into an average acceleration spectra by multiplying by twice the frequency squared and then taking the square root. We then measure the period at which the peak average acceleration spectra occurs. Geometrically, this occurs when the line with slope $\omega^{-2}$ with the highest amplitude is tangent to the energy spectra curve. The dashed lines in Figure 7 show such lines with slope $\omega^{-2}$. (To accommodate the situation where the peak acceleration spectra might be flat, we define this period to be the lowest period at which the peak occurs, to connect to the concept of corner frequency. This situation does not arise in our model spectra here, but may be relevant to other situations, such as the classical Brune $\omega^{-2}$ spectra [Brune, 1970]).

[36] The period of the peak average acceleration spectra, $T_{a}$, shows an interesting dependence on event size. This is plotted in Figure 8, with the lower dashed curves. The different curves again correspond to different frictional instabilities parameterized by $\gamma$. From Figure 8 we see the nontrivial nature of this period of the peak average acceleration spectra measurement. For the smallest events the period is set by the timescale $t_{0}$ of the dominant $\sigma_{t}$ stress drop mechanism. Changing $t_{0}$ changes $T_{a}$. Because this time-dependent regime of the friction is not very realistic, this is not a particularly interesting regime. We can, however, make $\sigma_{0}$ small enough so it affects only the very smallest events. Once the slip and velocity weakening stress drops begin to dominate things get much more interesting. For the moderate and large events, as we will see, a very important source parameter begins to control the period $T_{a}$. Note also that, interestingly, unlike the energy measurement, the period $T_{a}$ saturates for the largest events.

[37] Figure 9 shows our second principal result. We plot with solid lines, as in Figure 8, the period $T_{a}$ of the peak of the average acceleration spectra as a function of event rupture length. This is shown with the solid line in Figure 9 , with the circles corresponding to the slip weakening case and the pluses corresponding to the velocity weakening case. To connect to the source motions, we have plotted only half the period, $T_{a} / 2$, on Figure 9 [Boatwright and Choy, 1992] (this factor of $1 / 2$ is a rough factor relating the specific time function at a point on the fault lasting a given time to the dominant period of the emitted waves; it varies a bit with the specific time function. For example, Boatwright and Choy [1992] show that a symmetric triangle of duration $T$ has a corner frequency at $T / 2$.).

[38] On Figure 9 we also plot an additional set of points. For each individual event these points show the average slip duration of points on the fault plotted against the length of the event. That is, we plot the mean slip duration $\theta$ against rupture length $L$, where

$$
\theta=\frac{\int \Theta(\mathrm{v}) d t d x}{L},
$$

with $\Theta$ the heaviside step function, which is 1 when the fault is slipping $\left(\mathrm{v}=\partial U /\left.\partial t\right|_{y=0}>0\right)$ and 0 when stuck $(\mathrm{v}=0)$. Again, we use the two different symbols for the two different frictions, with circles for slip weakening and pluses for velocity weakening. Note, as with $T_{a}$, the somewhat

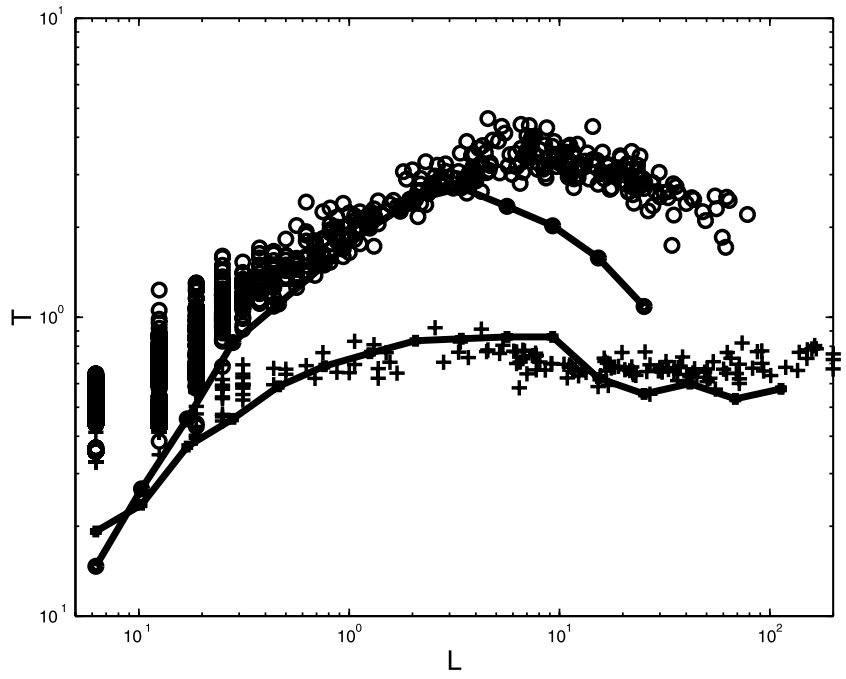

Figure 9. Comparison of the period of the peak average acceleration spectra for events of different sizes with the mean slip duration source parameter for different events. The solid lines are 1/2 times the period of the peak average acceleration spectra (see text for discussion of this factor of $1 / 2$ ). The individual points are the mean slip durations of individual events. Circles correspond to slip-weakening events $(\gamma=0.1)$, while pluses correspond to velocityweakening events $(\gamma=3)$.

surprising result that the mean slip duration decreases for the largest events, at least for the slip weakening case.

[39] As noted before, for the very smallest events the time-dependent stress drop $\sigma_{t}$ dominates the spectra, and $T_{a}$ is set by $t_{0}$. Thus $T_{a}$ falls well below the mean slip duration points. Once the slip- and velocity-weakening stress drop terms begin to dominate, however, we see that $T_{a}$ scales with $\theta$. The crossover of $T_{a}$ from $t_{0}$ scaling to $\theta$ scaling depends on the relative strength of $\sigma_{0}$; making $\sigma_{0}$ smaller pushes the crossover to smaller values of $L$. Because slipand velocity-weakening frictions are more realistic, this scaling with $\theta$ is the more relevant regime. It is particularly interesting that this corner frequency reflects a very natural and fundamental source parameter, the slip duration, and, moreover, that this relates to a topic of widespread interest and debate, the existence of slip pulses [Heaton, 1990; Beroza and Spudich, 1988; Perrin et al., 1995; Cochard and Madariaga, 1996; Langer et al., 1996; Beeler and Tullis, 1996; Day et al., 1998]. Further, we see an interesting magnitude dependence to this.

[40] For ruptures that propagate in uniform stress fields, classical crack solutions [Kostrov, 1964], as well as recent expanding pulse solutions [Nielson and Carlson, 2000] predict linear increases in the mean slip duration; our resolution is too limited to explore this scaling, but we do see an increase. For long narrow ruptures, however, the ruptures feel the width $W$ of the loaded seismogenic zone, and this scaling will break down; one then expects saturation. The somewhat surprising effect is that the mean slip duration not only saturates but decreases for the largest events, especially in the case of slip weakening. Evidently, the slip pulses of these events narrow relative to their maximum width, which occurs, we see from Figure 9, at 


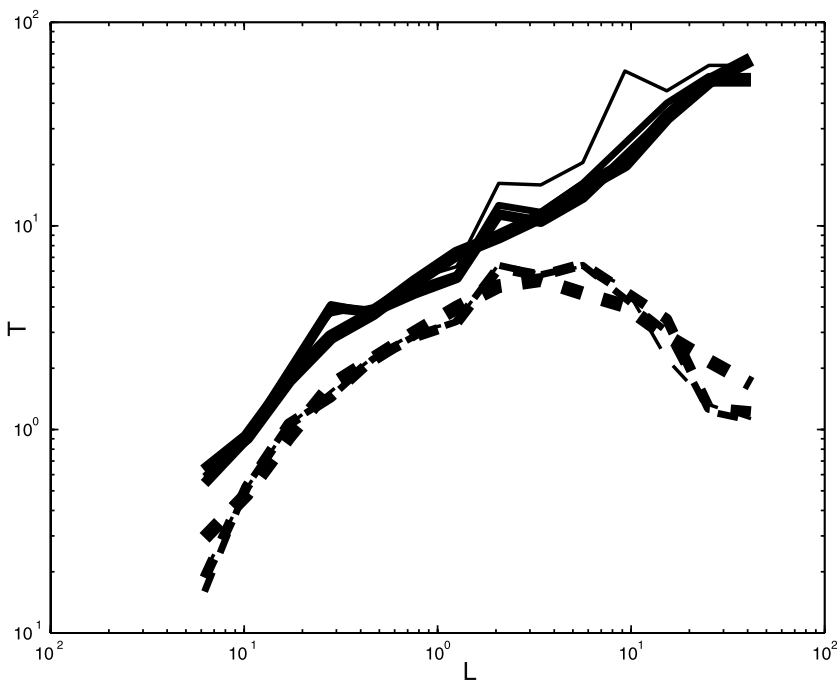

Figure 10. Two corner frequencies for spectra as a function of rupture length $L$. The different thickness curves correspond to different distances of the measurement surface from the fault plane, with thicker lines corresponding to larger values of $\Delta_{y}$. The values shown are $\Delta_{y}=1,2$, 4,6 , respectively. The top solid curves are the period of the peak energy density, while the bottom dashed curves are the period of the peak-averaged acceleration spectra.

$L$ of order a few $W$. After that the pulses break out into bilateral ruptures which narrow. This can all be seen in the time domain plot of the example large event shown earlier in Figure 4. What is key here is that even in the absence of the privileged view that Figure 4 affords in measuring velocities along the fault, we are able to observe these source effects in the frequency domain in the far field, an area accessible to standard observations!

\section{Discussion}

[41] A couple of other measurements support the results we have presented. One concerns the extrapolation of the measurements of the two corner frequencies to the far field. In the case of the small events, we are already in the far field, so this is not an issue. Figure 10 shows the same measurements as in Figure 8, made now for a fixed value of $\gamma$, and different values of $\Delta_{y}$. Evidently, our corners for even the large events are little affected by $\Delta_{y}$. Thus these corner frequency results appear to be valid for the far field.

[42] A second issue is a potential concern that the measurement of slip duration does not distinguish between fast slipping and slow slipping, and thus possibilities like long nucleation times or slow afterslip might bias the results shown in Figure 9, since the radiation is dominated by the fastest slip. This can be dealt with by an alternative to equation (8), through a measure of slip duration which weights time differently for fast and slow slip. One such alternate measure of slip duration arises from a dimensional argument. We define a radiative slip duration

$$
t_{\mathrm{v}}=\frac{\left(\left\langle\int \mathrm{v} d t\right\rangle\right)^{2}}{\left\langle\int \mathrm{v}^{2} d t\right\rangle},
$$

where the average (angle brackets) is over the rupture surface. This measure has dimensions of time and gives an effective slip duration for the parts of the fault that are slipping rapidly. We can make the same plot as in Figure 9 using this new timescale to measure the dynamically slipping part. Figure 11 shows this result. The effect at large lengths shows the large event effect is indeed real, with narrower pulses of concentrated slip developing.

[43] How many events might we need in order to see the mean spectral effects we have presented? Because the data sets are inherently limited, particularly for the rare longest events, it is useful to see what the data looks like with limited statistics. Figure 12 therefore shows the same type of plot as in Figure 8, except now we plot $T_{e}$ and $T_{a}$ in a disaggregated way for individual events; an event is represented by a line segment connecting the two measured corner periods. Evidently, the trends discussed earlier, the linear increase of $T_{e}$ with $L$, and the initial increase of $T_{a}$ with $L$ followed by a saturation for the largest $L$, are seen even for the noisy individual events. This disaggregation is additionally helpful as it shows the scatter in the data. Also, it is less sensitive than the mean plot to potential outliers.

[44] There is one last transformation we want to do in order to pose the model data in the way that renders it most clearly comparable to observations. Because the length of the rupture $L$ is often itself measured from $T_{e}$, there is a potential circularity in treating them as independent quantities in a plot. Therefore we use an alternative physical measure of the size of the event for the horizontal axis: the geometric moment $M$, representing the total slip on the fault. Figure 13 shows the same data as in Figure 12, only now ordered on the horizontal axis by $M$. The essential

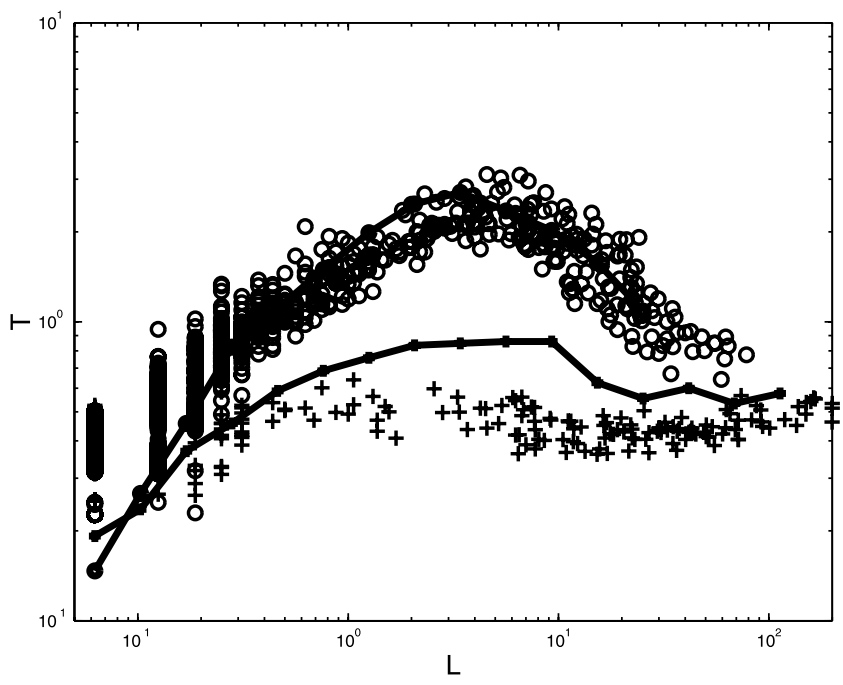

Figure 11. Comparison of the period of the peak average acceleration spectra for events of different sizes with the mean velocity-weighted radiative slip duration source parameter for different events. The solid lines are 1/2 times the period of the peak average acceleration spectra (see text for discussion of this factor of $1 / 2$ ). The individual points are the mean radiative slip durations of individual events. Circles correspond to slip-weakening events $(\gamma=0.1)$, while pluses correspond to velocity-weakening events $(\gamma=3)$. 

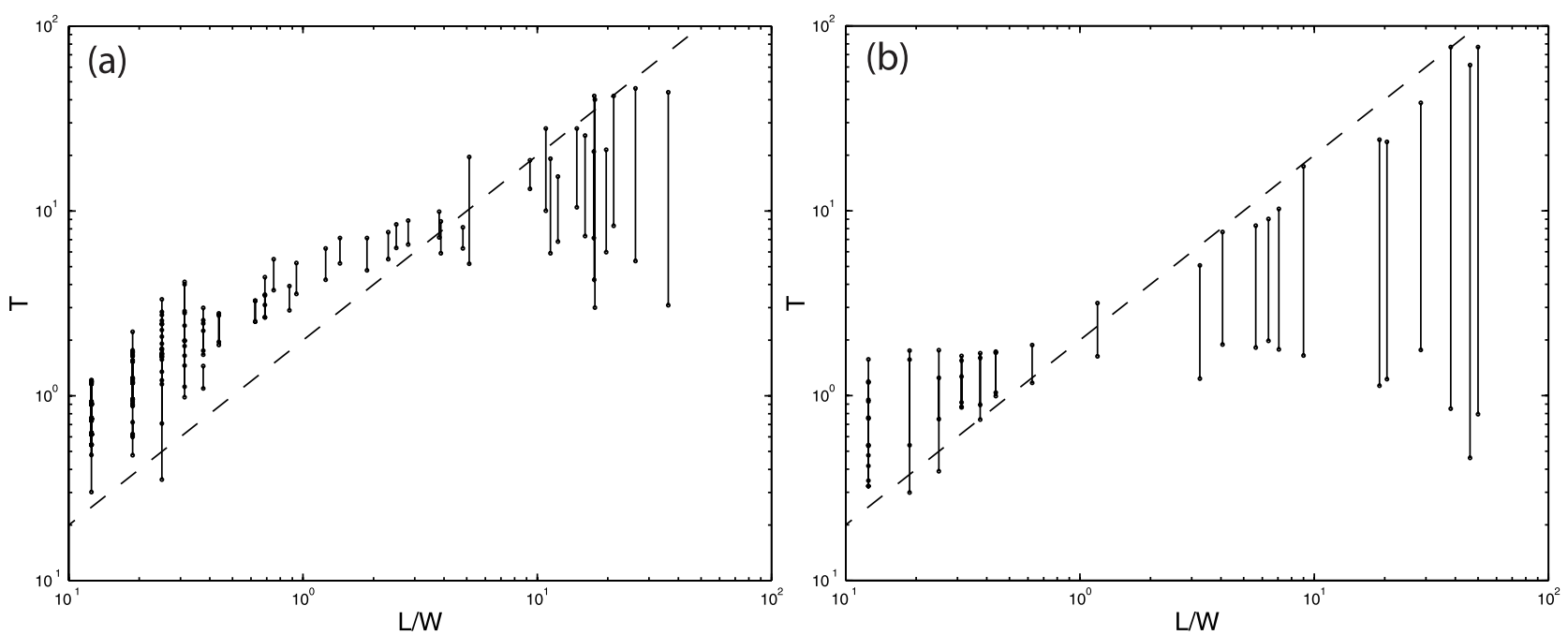

Figure 12. Two corner plots for individual events. Each line segment represents an individual event, with the upper value being the $\omega^{0}$ tangent peak energy density and the lower value being the $\omega^{-2}$ tangent peak average acceleration spectra. The horizontal axis is the rupture length of the event $L$. The dashed line shows a linear slope for comparison. Note that the trends evident in Figure 8 persist for the individual events. (a) Slip-weakening friction. (b) Velocity-weakening friction.

trend we have discussed is preserved. It will be extremely interesting to make these plots with real earthquakes, being careful to separate out different faulting styles of strike-slip and subduction zone events, so that different $W$ are not mixed.

\section{Appendix A: Friction}

[45] The friction we use has been explored previously [Shaw, 1995, 1997; Shaw and Rice, 2000]. The physical motivation goes back to Sibson [1973], whereby frictional sliding generates heat, thereby raising the pore fluid temperature and pressure, and thus decreasing the effective normal stress and friction. This gives frictional weakening from frictional heating. Our constitutive equations make a simple mathematical quantification and approximation of this effect. In addition to the physical motivation, they also have the advantage of spanning a range of frictional instabilities, from slip weakening in one limit to velocity weakening in another.

[46] Using an approximation [Shaw, 1997] of the full nonlinear case [Shaw, 1995], our constitutive equations for the friction $\Phi$ are

$$
\Phi=\phi\left(\frac{\partial S}{\partial t^{\prime}}, t^{\prime} \leq t\right) H\left(\frac{\partial S}{\partial t}\right)-\eta \nabla_{\|}^{2} \frac{\partial S}{\partial t} .
$$
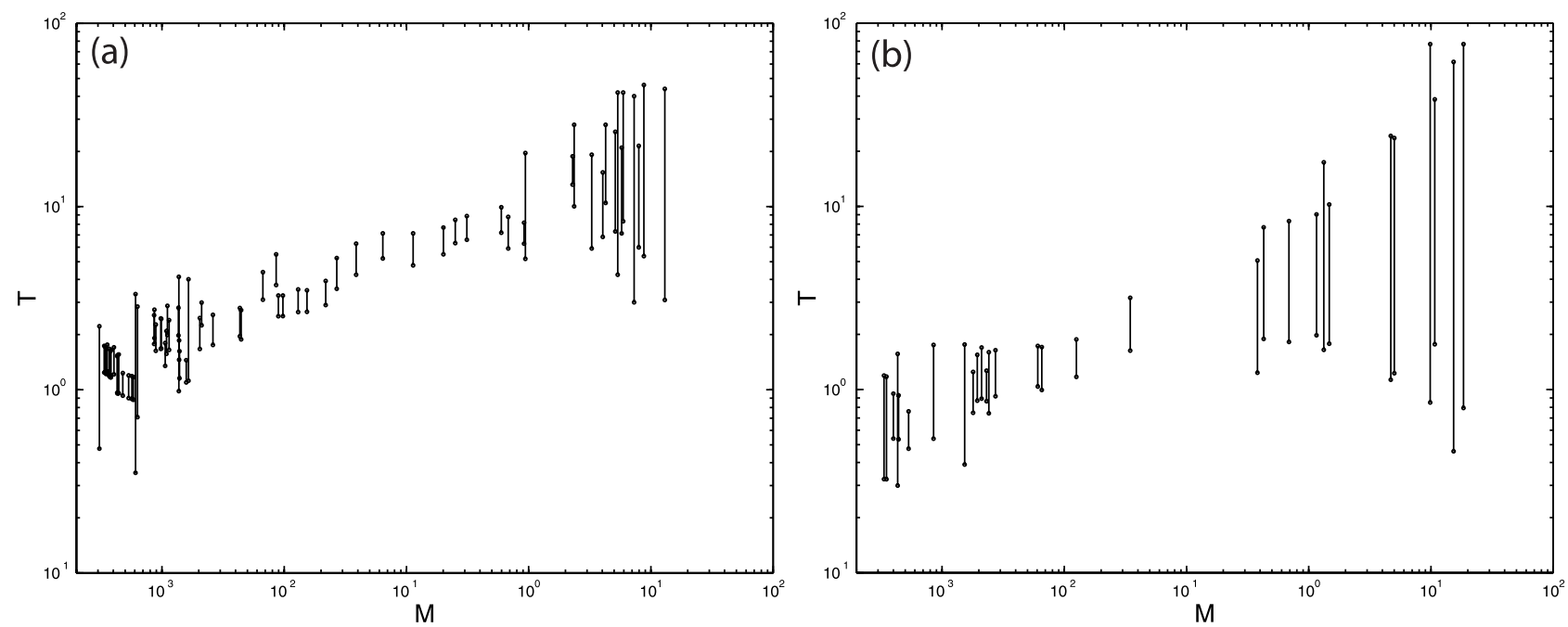

Figure 13. Two corner plots for individual events. Each line segment represents an individual event, with the upper value being the $\omega^{0}$ tangent peak energy density and the lower value being the $\omega^{-2}$ tangent peak average acceleration spectra. The horizontal axis is the moment of the event $M$. (a) Slip-weakening friction. (b) Velocity-weakening friction. 
Here $\partial S / \partial t=\partial U /\left.\partial t\right|_{y=0}$ is the slip rate on the fault, with $\phi$ depending on the past history of slip. The function $H$ is the antisymmetric step function, with

$$
H= \begin{cases}\frac{\partial \hat{S}}{\partial t} & \frac{\partial S}{\partial t} \neq 0 \\ |H|<1 & \frac{\partial S}{\partial t}=0\end{cases}
$$

where $\widehat{\partial S} / \partial t$ is the unit vector in the sliding direction. Thus $H$ represents the stick-slip nature of the friction, being multivalued at zero slip rate.

[47] The parameter $\eta$ is the strength of a viscous-like boundary dissipation, with $\nabla_{\|}^{2}=\partial^{2} / \partial x^{2}$ being the faultparallel Laplacian operator. This term is useful for giving stability to the smallest length scales [Langer and Nakanishi, 1993; Shaw, 1997].

[48] The history-dependent $\phi$ that we examine in this paper is given by

$$
\phi=\Phi_{0}-\frac{\alpha Q}{1+\alpha Q}-\Sigma
$$

with

$$
\frac{\partial Q}{\partial t}=-\gamma Q+\left|\frac{\partial S}{\partial t}\right|
$$

Here $\Phi_{0}$ is the threshold value of sticking friction, which, as long as it is large compared to the maximum friction drop, turns out the be an irrelevant parameter in the problem. The variable $Q$ is something like heat; it accumulates with increasing slip rate on the fault and dissipates on a timescale $1 / \gamma$. An equivalent integral solution of $Q$

$$
Q(t)=\int_{-\infty}^{t} e^{-\gamma\left(t-t^{\prime}\right)}\left|\frac{\partial S}{\partial t^{\prime}}\right| d t^{\prime}
$$

shows that when $1 / \gamma$ is large compared to the rupture timescale of unity, $Q$ is just the slip, while when $1 / \gamma$ is small, $Q$ rapidly reaches a steady state value of $1 / \gamma$ times the slip rate. Thus $\gamma$ controls the relative amount of slipweakening versus velocity-weakening effects [Shaw, 1995].

[49] The parameter $\alpha$ is the rate of weakening at small $Q$, which turns out to be a crucial parameter. It has dimensions of inverse length. The denominator $1+\alpha Q$ is used so as to saturate the drop in friction caused by this term at large $Q$, with the strength drop scaled to unity.

[50] The third term in the friction, $\Sigma$, describes the stress drop in going from sticking to sliding friction. We make a gross simplification of this term and, for simplicity, consider a $\Sigma$ which has two parts, one of which has the same form as the second term:

$$
\Sigma=\frac{\beta Q}{1+\beta Q / \sigma_{\beta}}+\sigma_{t}
$$

The $\sigma_{\beta}$ term gives a drop which weakens initially linearly in $Q$ with slope $\beta$, and then saturates to a constant stress drop $\sigma_{\beta}$ at large $Q$. The second term, $\sigma_{t}$, is a time-dependent nucleation term given by

$$
\sigma_{t}= \begin{cases}\sigma_{0} \frac{t-t_{s}}{t_{0}} & t-t_{s}<t_{0} \\ \sigma_{0} & t-t_{s} \geq t_{0},\end{cases}
$$

so that $\sigma_{t}$ increases linearly with time once the fault becomes unstuck, up to a maximum value $\sigma_{0}$ over a timescale $t_{0}$, and is reset to zero when the fault resticks. The time $t_{s}$ is measured from the last unsticking and is reset during an event if the fault resticks and then slips again.

[51] This $\Sigma$ term is a substantial simplification of what is likely to be happening in the Earth. A more realistic representation of this term would be the rate-and-state formulation [Dieterich, 1979; Ruina, 1983], but that formulation is much more expensive numerically, and it has been shown that many of the features of the model are insensitive to the details of the $\Sigma$ term, at least in the twodimensional models [Shaw and Rice, 2000].

\section{Appendix B: Radiating Boundary Condition}

[52] To allow for the very long events which spontaneously form in the model, we need a very long fault, of order hundreds of crust depths in length (thousands of kilometers, in dimensional units). We cannot afford numerically to have a bulk as wide as that so in order to avoid reflections off of the finite bulk domain, we need a radiating boundary condition away from the fault. We also want to study multiple events and avoid contamination from the radiation of previous events, particularly as previous large events, being orders of magnitude larger than the smallest events, would swamp the small events even for very tiny relative residuals. We have therefore applied a previously used technique, whereby between events the system is quenched to the static solution using the Dirichlet problem of fixed displacements on the fault boundary and on the far boundary [Shaw, 1997]

$$
\left.U\right|_{y=L_{y}}=\nu t
$$

The one difference is that now, during an event, we use radiating boundary conditions at the far boundary to make it transparent. Our innovation here is to modify the radiating boundary condition of [Clayton and Endquist, 1977] by recentering their finite difference formulation so that what previously worked off of an initially zero stress boundary now works off of an initially uniform displacement boundary (which is not in general a uniform stress boundary!). This is important, since when we let go of the boundary when the event starts so that the radiation will be able to pass through, we do not want it to start flapping spontaneously on its own. With this innovation we have a boundary which both sustains an initial stress, and is dynamically transparent. A judicious recentering of the Clayton and Endquist [1977] (equation (A2)) condition allows this. Specifically, we take their continuum radiating condition

$$
\frac{\partial^{2} U}{\partial t \partial y}+\frac{\partial^{2} U}{\partial t^{2}}-\frac{1}{2} \frac{\partial^{2} U}{\partial x^{2}}=0
$$



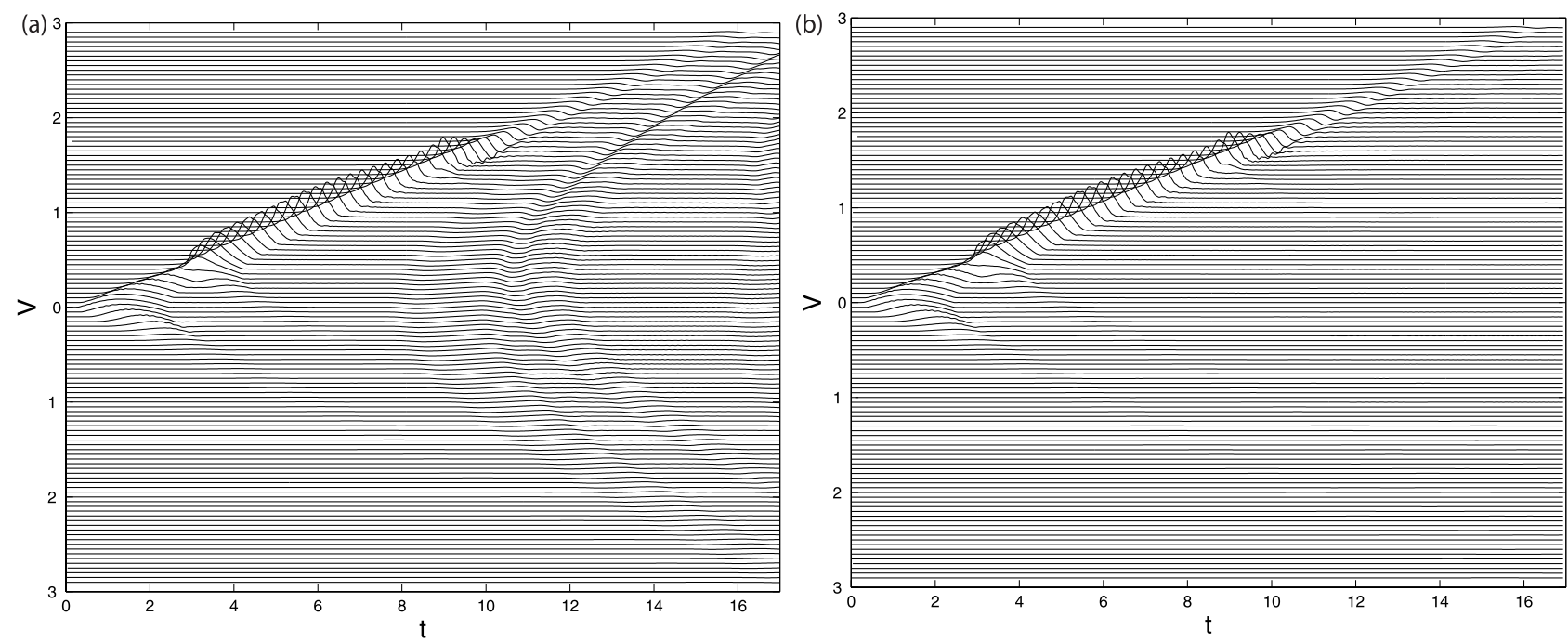

Figure B1. Illustration of utility of radiating boundary condition, by repeating same event using two different boundary conditions. The event is the same as in Figure 4. (a) Reflecting boundary condition. (b) Radiating boundary condition. Note absence now of reflection.

and recenter it compared to their finite difference approximation to take advantage of the constant displacement initial conditions; with this recentering, the curvature $\partial^{2} U / \partial x^{2}$ term is initially zero, along with the acceleration $\partial^{2} U / \partial t^{2}$ term, so $\partial^{2} U / \partial y \partial t$ will be zero until a wave impinges. Denoting as they do $n$ the integer index of the elements in the $x$ direction, and $k$ the integer index of the elements in the $y$ direction, so $U_{n k}(t) \equiv U\left(x=n \delta_{x}, y=k \delta_{y}, t\right)$, our new recentering of the finite difference approximation gives a time centered condition of

$$
\begin{aligned}
& \frac{1}{4 \delta y \delta t}\left[U_{n k+1}(t+1)-U_{n k+1}(t-1)-U_{n k-1}(t+1)\right. \\
& \left.\quad+U_{n k-1}(t-1)\right]+\frac{1}{\delta t^{2}}\left[U_{n k}(t+1)-2 U_{n k}(t)+U_{n k}(t-1)\right] \\
& \quad-\frac{1}{2 \delta x^{2}}\left[U_{n+1 k}(t)-2 U_{n k}(t)+U_{n-1 k}(t)\right]=0 .
\end{aligned}
$$

We can simplify this slightly in our case where we integrate explicitly in time, so the expression is no longer time centered, to get

$$
\begin{aligned}
& \frac{1}{2 \delta y}\left[U_{n k+1}(t+1)-U_{n k-1}(t+1)\right]=\frac{1}{2 \delta y}\left[U_{n k+1}(t)-U_{n k-1}(t)\right] \\
& \quad-\delta t\left[\frac{1}{2 \delta x^{2}}\left[U_{n+1 k}(t)-2 U_{n k}(t)+U_{n-1}(t)\right]\right. \\
& \left.+\frac{1}{\delta y^{2}}\left[U_{n k+1}(t)-2 U_{n k}(t)+U_{n k-1}(t)\right]\right]
\end{aligned}
$$

which is the new radiating boundary condition we use, with the acceleration having been replaced by the force, which is the Laplacian from the wave equation (1).

[53] Figures 3 and 4 illustrate this boundary's excellent behavior, being stationary before the radiating waves have hit, and nearly transparent as they encounter it. Figure B1 further illustrates this point by contrast, showing the top right panel as in Figure 4, and then repeating the run, but now with a fixed boundary condition; note the clear reflected waves in this latter case.

\section{Appendix C: Far-Field Low-Frequency Asymptotics}

[54] We estimate the low-frequency spectral shape of the radiation as follows. The Green's function for the wave equation in two dimensions is

$$
G\left(x, t ; x_{1}, t_{1}\right)= \begin{cases}0 & c\left(t-t_{1}\right)<r \\ \frac{1}{2 \pi c \sqrt{c^{2}\left(t-t_{1}\right)^{2}-r^{2}}} & c\left(t-t_{1}\right)>r\end{cases}
$$

where $r=\left|x-x_{1}\right|$. For low frequencies for timescales which are much longer than the timescale of the rupture process, the detailed shape of the source-time function is not important; so we consider the simplest source-time function, which is the box-car, having constant velocity $V_{1}$ over time $T$ and zero otherwise. Then

$$
\begin{aligned}
& U(x, t)=\int G\left(x, t ; x_{1}, t^{\prime}\right) V\left(t^{\prime}\right) d t^{\prime} \\
& = \begin{cases}0 & r<\left(t-t_{1}\right)<r \\
\frac{1}{2 \pi c} \int_{t_{1}}^{t_{1}-t-r / c} \frac{d t^{\prime} V_{1}}{\sqrt{c^{2}\left(t-t^{\prime}\right)^{2}-r^{2}}} & r<c\left(t-t_{1}\right)<r+c T \\
\frac{1}{2 \pi c} \int_{t_{1}}^{t_{1}+T} \frac{d t^{\prime} V_{1}}{\sqrt{c^{2}\left(t-t^{\prime}\right)^{2}-r^{2}}} & r+c T<c\left(t-t_{1}\right)\end{cases}
\end{aligned}
$$

$$
= \begin{cases}0 & c\left(t-t_{1}\right)<r \\ \frac{V_{1}}{2 \pi c^{2}} \log \frac{c\left(t-t_{1}\right)+\sqrt{c^{2}\left(t-t_{1}\right)^{2}-r^{2}}}{r} & r<c\left(t-t_{1}\right)<r+c T \\ \frac{V_{1}}{2 \pi c^{2}} \log \frac{c\left(t-t_{1}\right)+\sqrt{c^{2}\left(t-t_{1}\right)^{2}-r^{2}}}{c\left(t-t_{1}-T\right)+\sqrt{c^{2}\left(t-t_{1}-T\right)^{2}-r^{2}}} & r+c T<c\left(t-t_{1}\right)\end{cases}
$$


Taking the derivative with respect to time $t$ to get velocity gives

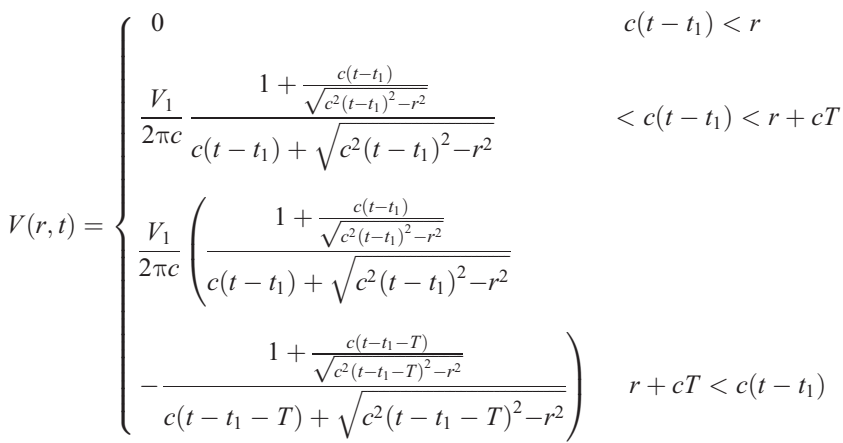

Rewriting in terms of the variable $\xi=c\left(t-t_{1}\right)-r$ and keeping only lowest order in $\xi / r$, an approximation good in the far field, gives

$$
V(r, \xi)= \begin{cases}0 & \xi<0 \\ \frac{V_{1}}{2 \pi c} \frac{1}{\sqrt{r \xi}} & 0<\xi<c T \\ \frac{V_{1}}{2 \pi c}\left(\frac{1}{\sqrt{r \xi}}-\frac{1}{\sqrt{r(\xi-c T)}}\right) & c T<\xi\end{cases}
$$

Calculating the Fourier transform of this gives

$$
\begin{aligned}
\hat{V}(\omega)= & \int_{0}^{\infty} V(r, t) e^{i \omega t} d t \\
= & \int_{0}^{T} \frac{V_{1}}{2 \pi c \sqrt{r}} \frac{1}{\sqrt{c t^{\prime}}} e^{i \omega t^{\prime}} d t^{\prime} \\
& +\int_{T}^{\infty} \frac{V_{1}}{2 \pi c \sqrt{r}}\left[\frac{1}{\sqrt{c t^{\prime}}}-\frac{1}{\sqrt{c t^{\prime}-c T}}\right] e^{i \omega t^{\prime}} d t^{\prime} \\
= & \frac{V_{1}}{2 \pi c^{3 / 2} \sqrt{r}} \int_{0}^{\infty}\left[1-e^{i \omega T}\right] \frac{e^{i \omega t^{\prime}}}{\sqrt{t^{\prime}}} d t^{\prime} \\
= & \frac{V_{1}}{2 c^{3 / 2} \sqrt{r}} \frac{\left(1-e^{i \omega T}\right)}{\sqrt{\omega}}
\end{aligned}
$$

where the last step came from contour integration. Thus we get

$$
\hat{V}(\omega)=-i e^{i \omega T / 2} \frac{V_{1}}{c^{3 / 2}} \frac{1}{\sqrt{r}} \frac{\sin \omega T / 2}{\sqrt{\omega}} .
$$

Taking the limit as $\omega \rightarrow 0$ gives

$$
|\hat{V}(\omega)|=\frac{V_{1} T}{2 c^{3 / 2}} \frac{\sqrt{\omega}}{\sqrt{r}}
$$

which is the asymptotic far-field low-frequency 2-D result we seek.

[55] Acknowledgments. I thank Jack Boatwright for a very thorough and constructive review and an anonymous reviewer for helpful comments. This work was supported by NSF grants EAR9909287 and EAR0087645,
USGS grant 1434HQ97GRO3074, and a grant from the Southern California Earthquake Center.

\section{References}

Aki, K., Scaling laws of seismic spectrum, J. Geophys. Res., 72, 1217, 1967.

Alford, R. M., K. R. Kelly, and D. M. Boore, Accuracy of finite difference modeling of the acoustic wave equation, Geophysics, 36, 834, 1974.

Atkinson, G. M., Earthquake source spectra in Eastern North America, Bull. Seismol. Soc. Am., 83, 1778, 1993.

Atkinson, G. M., and W. Silva, An empirical study of earthquake source spectra for California earthquakes, Bull. Seismol. Soc. Am., 87, 97, 1997.

Atkinson, G. M., and W. Silva, Stochastic modeling of California ground motions, Bull. Seismol. Soc. Am., 90, 255, 2000.

Atkinson, G. M., D. M. Boore, and J. Boatwright, Comment on "Earthquake source spectra in eastern North America" by R. A. Haddon, Bull. Seismol. Soc. Am., 87, 1697, 1997.

Beeler, N. M., and T. E. Tullis, Self-healing slip pulses in dynamic rupture models due to velocity dependent strength, Bull. Seismol. Soc. Am., 86, 1130, 1996.

Beroza, G. C., and P. Spudich, Linearized inversion for fault rupture behavior: Application to the 1984 Morgan Hill, California, earthquake, J. Geophys. Res., 93, 6275, 1988.

Boatwright, J., The seismic radiation from composite models of faulting, Bull. Seismol. Soc. Am., 78, 489, 1988.

Boatwright, J., and G. L. Choy, Acceleration source spectra anticipated for large earthquakes in northeastern North America, Bull. Seismol. Soc. Am., $82,660,1992$

Brune, J. N., Tectonic stress and the spectra of seismic shear waves from earthquakes, J. Geophys. Res., 75, 4997, 1970.

Clayton, R., and B. Endquist, Absorbing boundary conditions for acoustic and elastic wave equations, Bull. Seismol. Soc. Am., 67, 1529, 1977.

Cochard, A., and R. Madariaga, Complexity of seismicity due to highly rate-dependent friction, J. Geophys. Res., 101, 25,331, 1996.

Das, S., and B. V. Kostrov, An investigation of the complexity of the earthquake source time function using dynamic faulting models, J. Geophys. Res., 93, 8035, 1988.

Day, S., G. Yu, and D. J. Wald, Dynamic stress changes during earthquake rupture, Bull. Seismol. Soc. Am., 88, 512, 1998.

Dieterich, J. H., Modeling of rock friction, 1, Experimental results and constitutive equations, J. Geophys. Res., 84, 2161, 1979.

Haddon, R. A. W., Earthquake source spectra in eastern North America, Bull. Seismol. Soc. Am., 86, 1300, 1996.

Haddon, R. A. W., Reply to Comment on "Earthquake source spectra in eastern North America" by R. A. Haddon, Bull. Seismol. Soc. Am., 87, 1703, 1997.

Hartzell, S., and J. Brune, The Horse Canyon earthquake of August 2, 1975-Two-stage stress-release process in a strike-slip earthquake, Bull. Seismol. Soc. Am., 69, 1161, 1979.

Hartzell, S., S. Harmsen, A. Frankel, and S. Larsen, Calculation of broadband time histories of ground motion: Comparison of methods and validation using strong-ground motion from the 1994 Northridge earthquake, Bull. Seismol. Soc. Am., 89, 1484, 1999.

Haskell, N., Total energy and energy spectral density of elastic wave radiation from propagating faults, Bull. Seismol. Soc. Am., 54, 1811, 1964.

Heaton, T. H., Evidence for and implications of self-healing pulses of slip in earthquake rupture, Phys. Earth Planet. Inter, 64, 1, 1990.

Joyner, W., and D. Boore, On simulating large earthquakes by Green's function addition of smaller earthquakes, in Earthquake Source Mechanics, Geophys. Monogr. Ser., vol. 37, edited by S. Das, J. Boatwright, and C. H. Schlotz, p. 269, AGU, Washington, D. C., 1986.

Kostrov, B. V., Self-similar problems of propagation of shear cracks, J. Appl. Math. Mech., 28, 1077, 1964.

Langer, J. S., and H. Nakanishi, Models of rupture propagation, II, Two dimensional model with dissipation on the fracture surface, Phys. Rev. E, 48, 439, 1993.

Langer, J. S., J. M. Carlson, C. H. Myers, and B. E. Shaw, Slip complexity in dynamic models of earthquake faults, Proc. Natl. Acad. Sci. U. S. A., 93, 3825, 1996.

Madariaga, R., Dynamics of an expanding circular crack, Bull. Seismol. Soc. Am., 66, 639, 1976.

McGarr, A., Analysis of peak ground motion in terms of a model of inhomogeneous faulting, J. Geophys. Res., 86, 3901, 1981.

Miyatake, T., Numerical simulations of earthquake source process by a three-dimensional crack model, part I, Rupture process, part II, Seismic waves and spectrum, J. Phys. Earth, 28, 565, 1980.

Myers, C. H., B. E. Shaw, and J. S. Langer, Slip complexity in a crustal plane model of an earthquake fault, Phys. Rev. Lett., 77, 972, 1996. 
Nielson, S. B., and J. M. Carlson, Rupture pulse characterization: Selfhealing, self-similar, expanding solutions in a continuum model of fault dynamics, Bull. Seismol. Soc. Am., 90, 1480, 2000.

Olsen, K. B., R. Madariaga, and R. J. Archuleta, Three dimensional dynamic simulation of the 1992 Landers earthquake, Science, 278, 834, 1997.

Papageorgiou, A., and K. Aki, A specific barrier model for the quantitative description of inhomegeneous faulting and the prediction of strong ground motion, part I, Description of the model, Bull. Seismol. Soc. Am., 73, 693, 1983.

Perrin, G., J. R. Rice, and G. Zheng, Self-healing slip pulse on a frictional surface, J. Mech. Phys. Solids, 43, 1461, 1995.

Rice, J. R., Spatio-temporal complexity of slip on a fault, J. Geophys. Res., 98, 9885, 1993.

Ruina, A. L., Slip instability and state variable friction laws, J. Geophys Res., 88, 10,359, 1983.

Shaw, B. E., Frictional weakening and slip complexity on earthquake faults, J. Geophys. Res., 100, 18,239, 1995.

Shaw, B. E., Modelquakes in the two-dimensional wave equation, J. Geophys. Res., 102, 27,367, 1997.
Shaw, B. E., Far field radiated energy scaling in elastodynamic earthquake fault models, Bull. Seismol. Soc. Am., 88, 1457, 1998.

Shaw, B. E., and J. R. Rice, Existence of continuum complexity in the elastodynamics of repeated fault ruptures, J. Geophys. Res., 105, 23,791, 2000

Sibson, R. H., Interactions between temperature and pore fluid pressure during earthquake faulting and a mechanism for partial or total stress relief, Nature Phys. Sci., 243, 66, 1973.

Sokolov, V. Y., Empirical models for estimating Fourier-amplitude spectra of ground accleration in the Northern Caucasus (Racha Seismogenic Zone), Bull. Seismol. Soc. Am., 87, 1401, 1997.

Tumarkin, A. G., R. J. Archuleta, and R. Madariaga, Scaling relations for composite earthquake models, Bull. Seismol. Soc. Am., 84, 1279, 1994.

Zeng, Y., J. G. Anderson, and G. Yu, A composite source model for computing sythetic strong ground motions, Geophys. Res. Lett., 21, 725, 1994.

B. E. Shaw, Lamont-Doherty Earth Observatory, Columbia University, Route 9W, Palisades, NY 10964, USA. (shaw@1deo.columbia.edu) 